\title{
Comprensión de narraciones en niños de 5 y 6 años:
}

\section{Efectos de la memoria de trabajo verbal y la atención sostenida}

\section{Narrative comprehension in 5 and 6-year-old children:}

\section{Effects of working memory and sustained attention}

Juan Pablo Barreyroํㅜ, Jésica Formoso², Andrea Alvarez-Drexler ${ }^{3}$, Marina Leiman ${ }^{4}$, Rocío Fernández ${ }^{5}$, Alejandra Calero ${ }^{6}$, Julieta Fumagalli e Irene Injoque-Ricle ${ }^{8}$

${ }^{1}$ Doctor en Psicología. Investigador Adjunto de la Carrera del Investigador Científico y Tecnológico del Consejo Nacional de Investigaciones Científicas y Técnicas (CONICET). Instituto de Investigaciones, Facultad de Psicología, Universidad de Buenos Aires.

E-mail: jbarreyro@psi.uba.ar, jpbarreyro@gmail.com

${ }^{2}$ Doctora en Salud Mental. Becaria posdoctoral del Consejo Nacional de Investigaciones Científicas y Técnicas (CONICET). Instituto de Investigaciones, Facultad de Psicología, Universidad de Buenos Aires. jformoso@psi.ubar.ar

${ }^{3}$ Magister en Psicología Cognitiva y Aprendizaje de la Facultad Latinoamericana de Ciencias Sociales (FLACSO-UAM). Instituto de Investigaciones, Facultad de Psicología, Universidad de Buenos Aires. aldrexa@yahoo.com.ar

${ }^{4}$ Licenciada en Psicología. Becaria Estímulo. Instituto de Investigaciones, Facultad de Psicología, Universidad de Buenos Aires. marinaleiman@gmail.com

${ }^{5}$ Licenciada en Psicología. Becaria Estímulo. Instituto de Investigaciones, Facultad de Psicología, Universidad de Buenos Aires. rocio8923@hotmail.com

${ }^{6}$ Doctora en Psicología. Becaria posdoctoral del Consejo Nacional de Investigaciones Científicas y Técnicas (CONICET). Instituto de Investigaciones, Facultad de Psicología, Universidad de Buenos Aires. acalero@psi.uba.ar

${ }^{7}$ Doctora en Letras. Investigadora Asistente de la Carrera del Investigador Científico y Tecnológico (CONICET). Instituto de Lingüística, Facultad de Filosofía y Letras, Universidad de Buenos

Aires.julietafumagalli@filo.uba.ar

$8 \uparrow$ Doctora en Salud Mental. Investigadora Adjunta de la Carrera del Investigador Científico y Tecnológico (CONICET). Instituto de Investigaciones, Facultad de Psicología, Universidad de Buenos Aires.

Este trabajo fue realizado en el marco del proyecto UBACyT $2016 \mathrm{~N}^{\circ} 20020150200041 \mathrm{BA}$, otorgado al primer autor del trabajo.

En honor a la Dra. Irene Injoque-Ricle, gran compañera de trabajo y amiga.

Departamento de Procesos Psicológicos Básicos, Instituto de Investigaciones, Facultad de Psicología, Universidad de Buenos Aires (UBA).

Buenos Aires, Argentina

\section{Resumen}

Comprender un texto es una actividad cognitiva compleja que implica la construc- ción de una representación mental coherente en la memoria. Un proceso importante para ello es la generación de inferencias. La memoria de trabajo ha mostrado ser un factor 
cognitivo importante para explicar las diferencias en comprensión e inferencias, al igual que la capacidad de sostener la atención. El propósito del presente trabajo consiste en estudiar la relación entre la memoria de trabajo y la atención sostenida en la comprensión de narraciones en niños de 5 y 6 años. Para ello, se trabajó con una muestra de 100 niños, quienes escucharon tres textos narrativos y respondieron preguntas acerca de su contenido literal e inferencial, y además realizaron dos pruebas de memoria de trabajo y una de atención sostenida. Los resultados indican que los niños de 6 años muestran desempeños mejores y significativos en la comprensión de información literal y en la respuesta a preguntas de inferencia que los niños de 5 años. Además, el análisis de correlación mostró que las medidas de comprensión se hallan vinculadas a medidas de atención sostenida y a medidas de memoria de trabajo. El análisis de senderos sugiere que, en niños de 5 y 6 años, las mejoras logradas en la comprensión general como producto de la edad están mediadas, en parte, por la capacidad del niño para mantener la atención en la narración y almacenar temporalmente la información recibida en la memoria de trabajo mientras la escucha.

Palabras clave: Comprensión; Narraciones; Niños; Memoria de trabajo; Atención sostenida.

\section{Abstract}

Text comprehension involves the construction of a coherent mental representation, which requires the person to build bridges between the new information and the background knowledge. In adults, establishing associations between information provided by the text is frequently an automatic skill, while for children it implies an important cognitive effort. This ability develops gradually over time and is connected to the generation of inferences. Working memory and the ability to sustain attention are considered two crucial processes for comprehension. The purpose of this study is to analyze the role of working memory and sustained attention in the comprehension of narratives in 5 and 6-year-old children. The study included 100 children of 5 and 6 years of age, of both sexes, that participated with the informed consent of their parents. Three oral texts were narrated to the children by a professional storyteller. For each text, six questions were asked: three of literal content and three of inferential content. Additionally, two working memory tasks were administered with one task of sustained attention. Three analyses were performed: First, a correlation analysis, to study the associations between comprehension, working memory, and sustained attention measures. Then, a comparison analysis of comprehension, working memory, and sustained attention scores between ages 5 and 6. And finally, a path analysis to study the role of age, sustained attention, and working memory on comprehension. Spearman Rho analyses in the whole sample show that literal comprehension had a significant correlation with forward digit $\operatorname{span}(R h o=.37, p<.001)$, backward digit span $(R h o=.37, p<.001)$, and with the sustained attention task (Rho $=$ $-.37, p<.001)$. Inferences showed a significant correlation with forward digit span (Rho $=.36, p<.001)$, backward digit $\operatorname{span}($ Rho $=$ $.46, p<.001)$, and with the sustained attention task $(R h o=-.37, p<.001)$. Sustained attention measures showed a significant correlation with forward digit span $(R h o=-.34, p<$ $.001)$ and with backward digit $\operatorname{span}($ Rho $=$ $-.37, p<.001)$. The comparison analysis indicated significant differences between 5- and 6 -year-olds in sustained attention measures $\left(t_{(98)}=3.08, S E M=5.41, p<.01\right)$, literal comprehension $\left(t_{(98)}=4.05, S E M=0.59, p<\right.$ $.001)$, and inferences $(U=750.50, z=3.50$, $p<.001)$, but not in forward digit span $\left(t_{(98)}\right.$ $=1.43, S E M=0.34, p=.16)$ and backward digit $\operatorname{span}(U=1043.50, z=1.49, p=.14)$. A model of interrelation was proposed with age as independent variable, comprehension as dependent variable, and working memory and sustained attention as mediating variables, 
being comprehension, a latent factor formed by literal comprehension and inferences, and working memory another latent factor formed by forward digits and backward digits span. The path analysis showed a good fit of the data to the model $\left(\chi_{(5)}^{2}=1.93, p=.86 ; A G F I\right.$ $=.97, \mathrm{CFI}=.99, \mathrm{TLI}=.99, \mathrm{RMSEA}=.00)$. The analysis showed that 6-year-olds perform better than 5-year-olds in literal and inferential information, and in sustained attention, but not in working memory. The correlation analyses, on the other hand, indicated that comprehension measures are associated to working memory and sustained attention scores and the path analysis indicated that both working memory and sustained attention play a role in comprehension. This suggests that, in 5 and 6-year-olds, age has an effect on the comprehension of general information and the ability to generate inferences, but this effect is mediated, in part, by the child's ability to sustain attention on the narration and to temporarily store the information received while listening to it.

Keywords: Comprehension; Narratives; Children; Working memory; Sustained attention.

\section{Introducción}

La comprensión de un texto o un discurso se refiere a la construcción de una representación coherente acerca de su significado (Kintsch, 1998; Kintsch y van Dijk, 1978). Para lograr esta representación se deben establecer vínculos y conexiones entre la información presentada, y también entre ésta y el conocimiento previo. La construcción final será la base para el posterior recuerdo, reconocimiento o actividad que involucre el conocimiento adquirido del texto, ya sea escrito o narrado (Zwaan y Singer, 2003).

En adultos, el establecimiento de los vínculos y las conexiones entre la información provista por el texto es una habilidad frecuentemente automática, pero para muchos niños implica un esfuerzo cognitivo importante. De hecho, esta habilidad se desarrolla gradual- mente en el tiempo (Bourg, Bauer y van den Broek, 1997; Kendeou, van den Broek, White y Lynch, 2009), y está ligada a la generación de inferencias (Rapp, van den Broek y Kendeou, 2005; van den Broek, 1994). Por ejemplo, mientras que los niños pequeños son capaces de identificar las relaciones entre eventos concretos, los niños mayores son, a medida que crecen, más hábiles en identificar las relaciones entre eventos abstractos (Goldman, 1985; van den Broek, 1989). Los niños pequeños con frecuencia reconocen las conexiones entre los acontecimientos externos, mientras que los niños mayores y los adultos, normalmente, identifican las conexiones entre los eventos internos, como las metas y las emociones de los personajes.

La generación de inferencias se refiere a la activación de información que no se encuentra explícitamente enunciada en el texto, y esto permite dar mayor coherencia a la oración focal que se está leyendo o escuchando (Currie y Cain, 2015; van den Broek, Rohleder y Narváez, 1996). Las inferencias se identifican con representaciones mentales que se activan al tratar de comprender, sustituyendo, añadiendo e integrando información del texto o del conocimiento previo. Por ejemplo, si se lee la siguiente secuencia de frases: "El sol era muy cálido. El muñeco de nieve se derritió", se puede inferir que existe una relación causal entre ambas frases, aunque no esté explícitamente mencionada. Las inferencias permiten develar lo oculto de un texto, posibilitan leer entre líneas y hacer explícita en la mente la información implícita (Graesser, Leon y Otero, 2002; Leon y Peñalba, 2002; Molinari, Barreyro, Cevasco y van den Broek, 2011). Así, cualquier información que se extrae de un texto y que no está explícitamente expresada en él puede considerarse una inferencia (Graesser, 2007; McKoon y Ratcliff, 1992; van den Broek y Kendeou, 2008). Se ha observado que la capacidad de inferir relaciones semánticas varía significativamente con la edad: los niños más pequeños realizan inferencias identificando relaciones entre los 
acontecimientos individuales cercanos, pero con la edad conectan cada vez más grupos de eventos entre sí (van den Broek et al., 2005).

Las narraciones describen cómo los sucesos y las acciones de personajes, guiados por metas y propósitos internos, causan cambios en los eventos de una historia que transcurre en un tiempo y en un espacio (László, 2008; Stein, 1982). Las relaciones causales son un componente fundamental de la estructura de cualquier narración y tienen, en consecuencia, un importante papel en su comprensión, tanto que guían incluso el recuerdo de aquellas (Molinari et al., 2011). Desde temprana edad, los niños están expuestos a la estructura narrativa (Gorman, Bingham, Fiestas y Terry, 2016). Para comprender una narración, los niños no sólo deben comprender y codificar los eventos individuales de la historia, sino también conectar conceptualmente sus diferentes partes . Esto requiere, entre otras habilidades, la comprensión de la estructura de la prosa narrativa, la capacidad de hacer inferencias y el conocimiento del mundo sobre una gran variedad de situaciones y hechos (Graesser, Millis y Zwaan, 1997; van den Broek, 1994; van Dijk y Kintsch, 1983).

En psicolingüística, la memoria de trabajo y la capacidad de sostener la atención son definidas como dos procesos cruciales para la comprensión (van den Broek et al., 1996). Dada la linealidad del lenguaje, el sujeto debe tener la capacidad de poder sostener la atención sobre la tarea de comprensión (tanto para textos escritos como para textos narrados) y a su vez, la información comprendida debe ser retenida por la memoria de trabajo (McVay y Kane, 2012). La atención sostenida representa una función atencional básica que se puede definir como la capacidad de mantener el foco atencional sobre una tarea durante períodos prolongados de tiempo (Sater, Givens y Bruno, 2001). Esta capacidad permite focalizar la atención para poner en marcha procesos necesarios de almacenamiento y recuperación de información de la memoria -tanto de trabajo como de largo plazo- y resolver problemas
(Diamond y Goldman-Rakic, 1989). La memoria de trabajo es un concepto central en psicología y se refiere a un sistema temporal de memoria de capacidad limitada al servicio de tareas cognitivas complejas (Baddeley, 2010). En gran cantidad de investigaciones se ha mostrado que las diferencias individuales en memoria de trabajo explican las diferencias encontradas en la comprensión de textos y discursos (Barreyro, Cevasco, Burin y Molinari, 2012; Cain, Oakhill y Bryant, 2004; Currie y Cain, 2015; Finney, Montgomery, Gillam y Evans, 2014; Irrazabal, Saux y Burin, 2016; Van Dyke, Johns y Kukona, 2014).

La investigación de la comprensión de niños pequeños de 4 a 6 años se ha focalizado en estudiar el desarrollo de las habilidades básicas de la lectura, como la conciencia fonológica, el conocimiento de letras, y la adquisición del vocabulario (Kendeou et al., 2009; Lynch et al., 2008; Villalonga Penna, Padilla Sabaté y Burin, 2014). La comprensión de textos no se estudia bajo la modalidad de lectura, sino bajo modalidades de escucha oral de textos narrados (Bourg et al., 1997; van den Broek, 1997). Con este propósito, para obtener una medida válida de las habilidades de comprensión en niños preescolares la comprensión es evaluada en contextos no-textuales, empleando videos de dibujos animados (Kim, Kendeou, van den Broek, White y Kremer, 2008), libros de imágenes (Paris y Paris, 2003; Strasser y del Rio, 2013), narraciones de adultos o lecturas de adultos con imágenes (Gorman et al., 2016; Kendeou et al., 2009; Lynch et al., 2008; Silva y Cain, 2015). En todos ellos, la respuesta a preguntas de información literal e inferencial (Silva y Cain, 2015) o el recuerdo (Kim et al., 2008) ha sido la medida más empleada para evaluar el estado de la comprensión.

Investigaciones previas en niños pre-lectores (Currie y Cain, 2015; Paris y Lindauer, 1976; Strasser y del Rio, 2013) han estudiado el rol distintas variables cognitivas sobre la comprensión. 
Strasser y del Rio (2013) estudiaron cómo la memoria de trabajo, la profundidad y la amplitud del vocabulario, la teoría de la mente, el control inhibitorio y la atención sostenida incidían en el recuerdo de historias y la comprensión de narraciones sin palabras (imágenes). Los resultados mostraron que los modelos que explican el rendimiento en el recuerdo de historias y la comprensión de cuentos sin palabras son diferentes. Por un lado, el recuerdo solo estuvo influenciado por la memoria de trabajo y por el conocimiento acerca del vocabulario. La comprensión de narraciones sin palabras fue explicada en conjunto por aspectos ejecutivos (atención y control inhibitorio), vocabulario y los procesos de generación de inferencias y monitoreo. En la comprensión de historias por imágenes, la teoría de la mente no mostró efectos, se observó que el efecto de la amplitud del vocabulario está mediado por su profundidad y que el monitoreo y la generación de inferencias mediaban el efecto de la memoria de trabajo en la comprensión.

Paris y Lindauer (1976) realizaron una de las primeras investigaciones para estudiar el desarrollo de la habilidad de generar inferencias en niños. Presentaban a los niños una serie de oraciones que podían incluir una inferencia elaborativa (instrumental) de manera implícita o explícita (por ejemplo, en contexto explícito: "La maestra corta la carne con un cuchillo", y en contexto implícito: "La maestra corta la carne", luego se presentaban palabras clave (por ejemplo: cuchillo) y se solicitaba al niño el recuerdo de la oración. Los niños preescolares y escolarizados mostraron el mismo desempeño en el recuerdo de la oración de contexto explícito frente a la palabra clave, pero en el contexto implícito, solo los niños escolarizados recordaban correctamente la oración frente a la palabra clave. Este resultado indica una mejora en la capacidad de generar inferencias elaborativas en los niños escolarizados.

Currie y Cain (2015) estudiaron la relación entre la memoria de trabajo y el conocimiento del vocabulario en la generación de inferencias. El estudio se realizó en una muestra de niños de 5-6 años (preescolares), y 7-8 y 9-10 años (escolares), en las que evaluaron la generación de inferencias globales y locales, y midieron el conocimiento del vocabulario necesario para las historias y la capacidad verbal de la memoria de trabajo. A nivel general, los resultados mostraron que las diferencias en la edad de los participantes explicaban las mejoras obtenidas en preguntas de inferencias (local y global). En relación con la memoria de trabajo y el vocabulario, la memoria de trabajo mostró asociaciones significativas con el conocimiento del vocabulario, como también con la generación de inferencias. Los análisis de regresión indicaron que el conocimiento del vocabulario incide fuertemente sobre la generación de inferencias, como así también, pero en menor medida, la memoria de trabajo.

$\mathrm{Si}$ bien existe un número importante de investigaciones en relación con las habilidades de comprensión en niños, en la actualidad es incipiente la investigación en desarrollo de la comprensión durante la edad preescolar y los primeros años de la escuela primaria, pero es menor la cantidad vinculada a otras habilidades cognitivas (Kendeou et al., 2009; Lynch et al., 2008). El objetivo de este trabajo es estudiar la incidencia de la memoria de trabajo verbal y la capacidad de sostener la atención en la comprensión de narraciones (empleando medidas de comprensión de la información literal e inferencias) en niños de 5 y 6 años de edad.

\section{Método}

\section{Participantes}

La muestra estuvo compuesta por un total de 50 niños de 5 años de ambos sexos (27 mujeres y 23 varones) con una media de edad de 63.45 meses $(D E=2.89)$ y 50 niños de 6 años de edad también de ambos sexos (23 mujeres y 27 varones) con una media de edad 
de 76.20 meses $(D E=3.29)$. Los niños asistían a un colegio privado de nivel socioeconómico medio de la Ciudad Autónoma de Buenos Aires, Argentina (información brindada por las autoridades del colegio) y participaron de la experiencia con el consentimiento escrito de los padres, a los que se les informó en una reunión las características del estudio, se les aseguró la confidencialidad de la información y el anonimato de la participación. Fueron excluidos del estudio niños con dificultades de aprendizaje, déficits auditivos o del lenguaje y trastornos neurológicos o psiquiátricos. Esta información fue brindada por los padres y los miembros de la institución educativa.

\section{Instrumentos}

\section{Cuestionario de comprensión (ad hoc)}

Se utilizaron tres textos narrativos para niños analizados a partir del modelo de red causal (Suh y Trabasso, 1993; van den Broek, Risden y Husebye-Hartmann, 1995). El modelo de red causal está centrado en analizar la estructura causal de las narraciones y parte de la base que la representación construida en memoria para una narración incluye las relaciones causales entre cada suceso y aquellos otros que, en conjunto, condujeron a su ocurrencia (Suh y Trabasso, 1993). La identificación de cada relación causal deriva de la aplicación de una prueba contrafáctica relativa a la necesidad de un evento en relación con otro en las circunstancias de la historia. La prueba tiene la siguiente forma: si A no hubiera ocurrido, entonces B tampoco habría ocurrido. El juicio acerca de si este enunciado contrafáctico es verdadero en las circunstancias de la historia permite decidir si A es la causa o una condición de B (van den Broek, Risden y Husebye-Hartmann, 1995). Este análisis se realiza sobre cada cláusula de los textos y permitió detectar ciclos de lectura que requerían de la generación de inferencias, al necesitar de información adicional proveniente del conocimiento del lector para una mayor explicación. Una vez detectadas las cláusulas oracionales que requieren de inferencias, se confeccionó un cuestionario con seis preguntas para cada texto con el objetivo de evaluar la comprensión de información literal y la generación de inferencias. Los textos utilizados fueron: "La sorpresa de Nandi" de Eileen Browne (1996), "Hipo no nada" de Pablo Bernasconi (2007) y la fábula de Esopo, el zorro y la cigüeña adaptada por Ran-hee Jung (2009), que tienen una extensión de entre 191 y 310 palabras. Los cuestionarios contenían seis preguntas de cada uno, tres que evaluaban la comprensión de información literal del texto y tres que evaluaban la generación de inferencias. Por ejemplo, para el texto el zorro y la cigüeña, como pregunta literal, se preguntó al niño: "¿Qué le dio de comer el zorro a la cigüeña?". La respuesta correcta consistía en recuperar la idea de que el zorro le dio de comer sopa a la cigüeña. La pregunta inferencial fue: "¿Por qué la cigüeña invita a cenar al zorro?". La respuesta correcta consistía en recuperar la idea de que la cigüeña lo invita al zorro para vengarse, en desquite o revancha de no haber podido comer la comida que el zorro le sirvió.

\section{Tarea de memoria de trabajo}

Se utilizó la prueba de dígitos de la Batería de Evaluación para niños de Kaufman (K-abc, Kaufman Assessment Battery for Children, 1983). Las pruebas tienen como objetivo medir la capacidad de almacenamiento de información verbal de la memoria de trabajo y la capacidad de almacenamiento y procesamiento concurrente de información de la memoria de trabajo, para lo cual se les administraron dos pruebas. En la primera (dígitos en orden directo) se le presentaban al niño series de dígitos que debían repetir en voz alta y en forma inmediata, en el orden exacto en el que se le habían presentado, y en la segunda (dígitos en orden inverso) se le presentaba una secuencia de dígitos que debían decir en voz alta y en forma inmediata, pero en el orden 
inverso (de atrás hacia adelante) al presentado. Si bien la tarea no muestra baremos para la población argentina, se la empleó por ser una prueba estándar para la evaluación de la memoria de trabajo pensada para niños preescolares, ya que no se encuentran pruebas de memoria de trabajo para niños pequeños adaptadas. Se utilizó, para cada prueba, el puntaje bruto que se obtiene de la suma de la cantidad de ítems recordados correctamente.

\section{Tarea de atención sostenida}

Se administró la prueba casita de animales del WPPSI (Test de inteligencia para preescolares, Wechsler, 1998). Esta tarea tiene como objetivo medir la velocidad de procesamiento de la información en niños, pero se ha observado que muestra buenos indicadores de validez para la evaluación de la atención sostenida y focalizada (Soprano, 2009) y prescinde del conocimiento de la forma visual de los números y las letras. La tarea consiste en la presentación de un tablero con dibujos de animales y cilindros de madera de diferente color, cada color correspondiente a un animal, donde el niño debe aparear la mayor cantidad de animales con su cilindro correspondiente durante 5 minutos. Se evalúa el tiempo total en la resolución de la tarea en segundos. Se utilizó como medida el puntaje bruto, resultado de la medición del tiempo en segundos en la resolución de la tarea, a partir de la idea de que un mejor desempeño se asocia a un menor tiempo de resolución.

\section{Procedimiento}

La administración de los instrumentos se realizó de forma individual en dos sesiones de aproximadamente 30 minutos cada una. En la primera sesión se presentaron al niño las tareas de evaluación cognitiva (de atención sostenida y memoria de trabajo) y en la segunda, se administraron textos narrados video-filmados por una narradora y el cuestionario de preguntas sobre cada uno de los textos de a uno por vez. El niño escuchaba una historia y luego respondía a las preguntas. A continuación, escuchaba la segunda historia y respondía a sus preguntas, del mismo modo que pasaba a la siguiente historia. Para que no incidiera el efecto de la presentación de las historias, se contrabalanceó el orden de esa presentación. Toda la tarea se llevó a cabo en un ambiente libre de ruidos, dentro de la institución educativa.

\section{Análisis de datos}

En primer lugar, se analizó la distribución de las puntuaciones de comprensión (comprensión de la información literal e inferencias), memoria de trabajo y atención sostenida, obteniendo así los estadísticos descriptivos. En segundo lugar, se realizó un análisis de correlación, tanto general como por edades, con el fin de medir el nivel de asociación entre las variables. En tercer lugar, se realizó un análisis de comparación de medias para determinar si existen diferencias en el rendimiento en las distintas tareas en los dos grupos de niños. En cuarto lugar, se llevó a cabo un análisis de senderos a partir del método de ecuaciones estructurales, proponiendo un modelo de relación entre las variables analizadas. El modelo propone que la edad en meses de los participantes incide de forma directa en la capacidad de la memoria de trabajo verbal, en la atención sostenida y en la comprensión. La memoria de trabajo verbal y la atención sostenida inciden de forma directa en la comprensión de narraciones, y, a su vez, están asociadas entre sí. Para este análisis se utilizó la estimación de máxima verosimilitud como matriz entre las medidas como input para el análisis de datos (Arbuckle, 2014), y se utilizaron los índices de ajuste, siguiendo recomendaciones y convenciones (Hu y Bentler, 1998, 1999). Los índices de ajuste elegidos han sido: $\chi^{2}$ (como prueba de bondad de ajuste del modelo propuesto a los datos), AGFI (Adjusted Goodness of Fit Index), CFI (Comparative Fit Index), TLI (TuckerLewis Index) y RMSEA (Root Mean Square Error of Aproximation). 


\section{Resultados}

En primer lugar se obtuvieron los estadís- ticos descriptivos, de distribución y de ajuste a una distribución normal. En la Tabla 1 se pueden observar los estadísticos analizados.

\section{Tabla 1}

Estadísticos descriptivos.

\begin{tabular}{c|c|c|c|c|c|c|c|c} 
& $\mathbf{M}$ & $\mathbf{D E}$ & Mín. & Máx. & As. & $\mathbf{C u .}$ & KS. & $\mathbf{p}$ \\
\hline Dígitos Directo & 5.48 & 1.69 & 2 & 10 & .21 & -.21 & 1.22 & .10 \\
Dígitos Inverso & 2.68 & 1.08 & 0 & 5 & .04 & .29 & 1.94 & .00 \\
Casita de Animales & 88.79 & 28.19 & 52 & 220 & 1.77 & 5.82 & 1.06 & .21 \\
Comprensión Literal & 11.52 & 3.19 & 4 & 17 & -.42 & -.55 & 1.10 & .18 \\
Inferencias & 6.81 & 3.38 & 0 & 16 & .43 & -.16 & 1.55 & .02
\end{tabular}

Como se puede observar en la Tabla 1, la distribución de las puntuaciones de la prueba de ordenamiento de dígitos en orden inverso y la respuesta a preguntas inferenciales de los textos muestran distribuciones alejadas significativamente de la distribución normal asintótica. Por esta razón, para los análisis subsiguientes se emplearon pruebas no paramétricas para trabajar dichas puntuaciones y se realizó la transformación a su logaritmo natural para llevar a cabo los análisis por ecuaciones estructurales.

A continuación, y con el propósito de estudiar las relaciones entre la memoria de trabajo, la capacidad de sostener la atención y el desempeño en la respuesta a preguntas acerca del contenido literal e inferencial del texto, se llevaron a cabo correlaciones empleando el estadístico de rangos de Spearman (Rho) en el grupo en general y luego por cada una de las edades. En la Tabla 2, se muestran las correlaciones generales y en la Tabla 3, las correlaciones para los niños de 5 y 6 años.

Tabla 2

Correlaciones (Rho) en la muestra general.

\begin{tabular}{c|c|c|c|c} 
& $\mathbf{1 .}$ & $\mathbf{2 .}$ & $\mathbf{3 .}$ & $\mathbf{4 .}$ \\
\hline 1. Dígitos Directo &. & & & \\
2. Dígitos Inverso & $.57^{* *}$ &. & & \\
3. Casita de Animales & $-.31^{* *}$ & $-.28^{* *}$ &. & \\
4. Comprensión Literal & $.37^{* *}$ & $.37^{* *}$ & $-.34^{* *}$ &. \\
5. Inferencias & $.36^{* *}$ & $.46^{* *}$ & $-.37^{* *}$ & $.54^{* *}$
\end{tabular}

$* * \mathrm{p}<.01$ 
Tabla 3

Correlaciones (Rho) en la muestra de niños de 5 y 6 años.

\begin{tabular}{c|c|c|c|c|c} 
& $\mathbf{1 .}$ & $\mathbf{2 .}$ & $\mathbf{3 .}$ & $\mathbf{4 .}$ & $\mathbf{5 .}$ \\
\hline 1. Dígitos Directo &. & $.48^{* *}$ & $-.28^{*}$ & $.38^{* *}$ & $.40^{* *}$ \\
2. Dígitos Inverso & $.62 * *$ &. & $-.29 *$ & $.44 * *$ & $.47 * *$ \\
3. Casita de Animales & $-.32 *$ & -.27 &. & $-44 * *$ & $-.29 *$ \\
4. Comprensión Literal & $.31 *$ & .25 & -.16 &. & $.57 * *$ \\
5. Inferencias & $.31 *$ & $.44 * *$ & $-.37 * *$ & $.47 * *$ &.
\end{tabular}

Nota: Las correlaciones de los niños de 5 años se muestran por encima de la diagonal y las correlaciones de los niños de 6 años se muestran por debajo de la diagonal.

$* \mathrm{p}<.05 . * * \mathrm{p}<.01$

Como se puede observar en la Tabla 2, el análisis muestra que hay correlación significativa de mediana intensidad e importante entre la respuesta a preguntas acerca del contenido literal del texto y las inferenciales $(R h o=.54$, $p<.001)$. La respuesta a preguntas literales se asocia significativamente con el desempeño en la prueba de dígitos en orden directo $(R h o=.37$, $p<.001$ ), con la prueba dígitos en orden inverso $(R h o=.37, p<.001)$ y la prueba de casita de animales $(R h o=-.34, p<.001)$. La respuesta a preguntas acerca del contenido inferencial de los textos mostró asociaciones significativas con dígitos directos $(R h o=.36, p<.001)$, con dígitos inverso $(R h o=.46, p<.001)$ y con casita de animales $(R h o=-.37, p<.001)$.

El análisis de las correlaciones en el grupo de niños de 5 años muestra que la respuesta a preguntas literales se asocia significativamente al desempeño de la prueba de dígitos directos $(R h o=.37, p<.01)$, la prueba de dígitos inverso $(R h o=.44, p<.01)$ y la prueba de casita de animales $(R h o=-.44, p<.01)$. El mismo resultado se obtiene en la relación con el desempeño de preguntas acerca de inferencias con dígitos directos $(R h o=.40, p<.01)$, con dígitos inverso $(R h o=.47, p<.01) \mathrm{y}$ casita de animales $(R h o=$ $-.29, p=.04)$. El análisis en las puntuaciones de los niños de 6 años muestra que el desempeño en las preguntas literales se encuentra asociado únicamente a la prueba de dígitos directos (Rho $=.31, p=.03$ ); en cambio, el desempeño en preguntas de inferencia muestra una asociación con las tres pruebas: dígitos directos $(R h o=.31$, $p=.03)$, dígitos inverso $(R h o=.44, p<.01) \mathrm{y}$ casita de animales $(R h o=-.37, p<.01)$.

A continuación, se realizó un análisis para comparar el desempeño de los niños de 5 y 6 años en las medidas estudiadas. En la Tabla 4 pueden observarse los estadísticos descriptivos de las medidas de dígitos directos, dígitos inversos, casita de animales, comprensión literal e inferencias. 
Tabla 4

Estadísticos descriptivos por edad.

Dígitos Directo (P. Máx. $=14)$
Dígitos Inverso (P. Máx. $=12$ )

Casita de Animales (P. Máx. $=300$ )

Comprensión Literal (P. Máx. $=18)$

Inferencias (P. Máx. = 18)

\begin{tabular}{|c|c|c|c}
\multicolumn{2}{|c|}{5 años } & \multicolumn{2}{c}{6 años } \\
$M$ & DE & M & DE \\
\hline 5.24 & 1.65 & 5.72 & 1.72 \\
2.50 & 1.06 & 2.86 & 1.09 \\
97.12 & 30.89 & 80.46 & 22.57 \\
10.32 & 3.33 & 12.72 & 2.55 \\
5.62 & 2.88 & 8.00 & 3.45
\end{tabular}

El análisis indica que los niños de 5 y 6 años no muestran diferencias en su desempeño en la prueba de dígitos en orden directo $\left(t_{(98)}=1.43, S E M=.34, p=.16\right)$ y tampoco en la prueba de dígitos en orden inverso $(U$ $=1043.50, z=1.49, p=.14)$. Se observan diferencias estadísticamente significativas en la prueba de casita de animales $\left(t_{(98)}=3.08\right.$, $S E M=5.41, p<.01)$, con menores tiempo (mejor desempeño) para los niños de 6 años en comparación con los niños de 5 años. En relación con las medidas de comprensión, los niños de 6 años muestran un desempeño significativamente mayor a los niños de 5 en la respuesta a preguntas literales del texto $\left(t_{(98)}=4.05, S E M=.59, p<.001\right)$ y también a preguntas inferenciales $(U=750.50, z=3.50$, $p<.001)$.

El análisis de ecuaciones estructurales realizado muestra que el modelo propuesto para los datos presenta excelentes indicadores de ajuste a los datos empíricos obtenidos con un despreciable error de aproximación (ver Figura 1) $\left(\chi_{(5)}^{2}=1.93, p=.86 ; A G F I=.97\right.$, CFI $=.99$, TLI $=.99$, RMSEA $=.00)$ y la varianza explicada de las variables incluidas en el modelo sobre la variable dependiente (Comprensión) es alta en torno al $61 \%\left(R^{2}=\right.$ $.61)$.

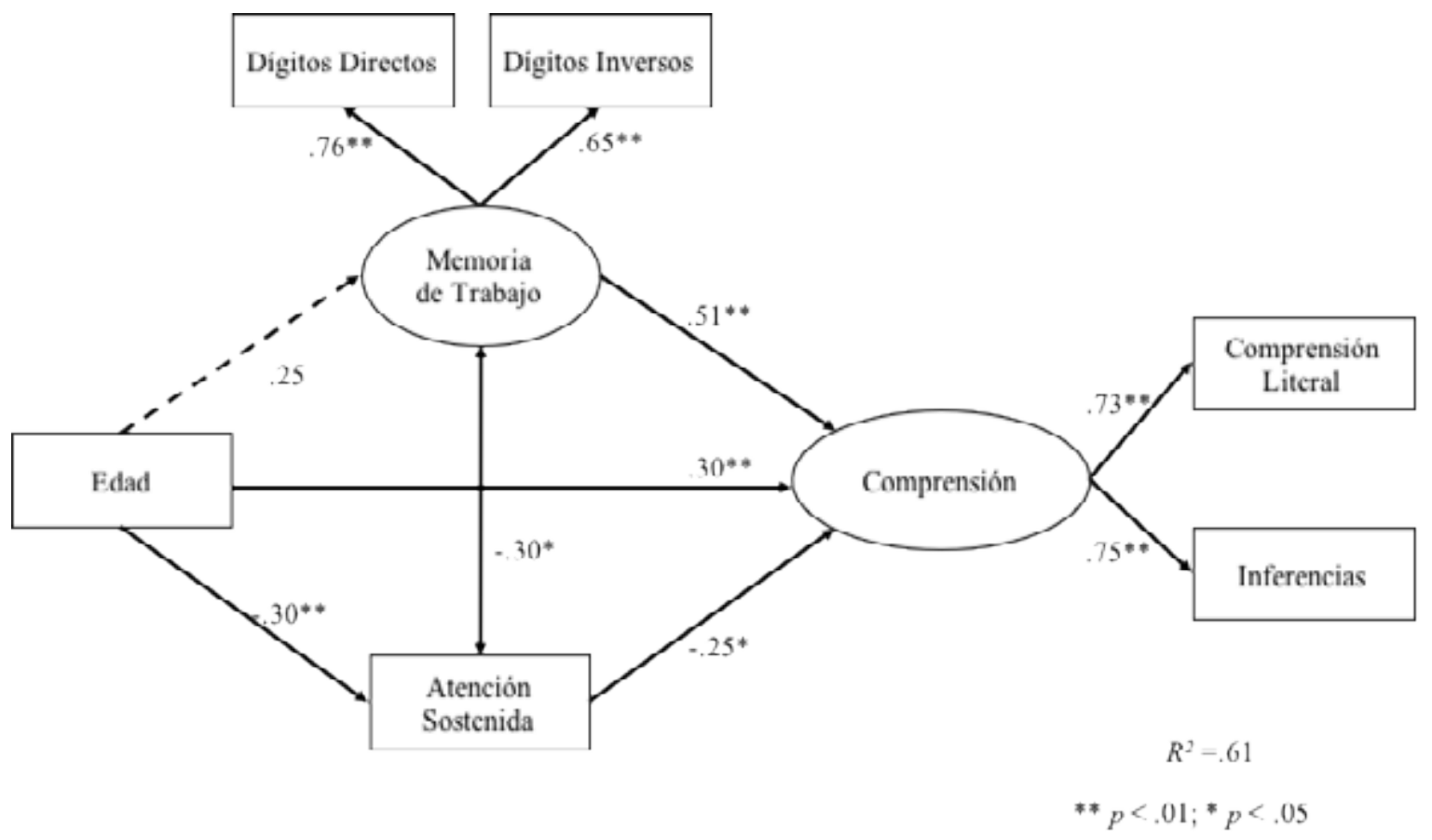

Figura 1. Modelo de relación entre la edad, la memoria de trabajo, la atención sostenida y la comprensión. 
Al observar los pesos de regresión, se observa que la edad de los participantes (en meses) tiene un efecto directo sobre la tarea de atención sostenida $(\beta=-.30, p<.01) \mathrm{y}$ sobre la comprensión de las narraciones $(\beta=$ $.30, p<.01)$, no así sobre la capacidad general de la memoria de trabajo $(\beta=.21, p=.10)$. La memoria de trabajo mostró un efecto significativo importante sobre la comprensión $(\beta=$ $.50, p<.001)$, mientras que la atención sostenida, si bien mostró un efecto significativo $(\beta=-.25, p=.03)$, ha sido menor. En cuanto al efecto indirecto de la edad mediado por la atención sostenida también fue significativo $(\beta=.18, p=.02)$. Por lo tanto, es de destacar que la edad mostró un efecto de mediación parcial a través de la atención sostenida, ya que presenta efectos directos sobre la comprensión, pero también indirectos. En cuanto a la relación entre la capacidad de la memoria de trabajo y la capacidad de sostener el foco de atención, la relación observada también fue significativa $(r=.30, p=.03)$.

\section{Discusión}

El objetivo del presente trabajo consistió en estudiar el efecto de la memoria de trabajo verbal y la capacidad de sostener la atención en la comprensión de narraciones orales teniendo en cuenta la edad de los niños (de 5 y 6 años). Con este fin, se administraron tareas de comprensión de narraciones, en donde los niños oían dos cuentos previamente videograbados por una narradora experta y respondían a preguntas acerca del contenido literal (información explícitamente presentada en el texto) e inferencial del texto (información implícita). Los niños también completaban una tarea que implicaba su capacidad para poder sostener su atención sobre una tarea determinada (Casita de Animales, Wechsler, 1998) y dos tareas de memoria de trabajo, una que implicaba su capacidad de poder retener información verbal por un breve periodo de tiempo (Recuerdo de dígitos en orden directo, Kaufman y Kaufman, 1983), y otra que implicaba su capacidad para retener y manipular información verbal en un breve periodo de tiempo (Recuerdo de dígitos en orden inverso, Kaufman y Kaufman, 1983).

Los resultados del análisis de correlación en el total de la muestra indicaron que la comprensión de información literal, esto es de la información explícitamente enunciada en los textos narrados, mostraban una asociación con la capacidad de retención de información en memoria de trabajo, como también con la capacidad de almacenamiento y procesamiento concurrente de la memoria de trabajo y la capacidad de poder sostener el foco de atención sobre una tarea determinada en un período breve de tiempo. Este resultado se observó en la muestra de niños de cinco años; en cambio en la muestra de los niños de seis años se observó que la comprensión de la información literal se encontraba asociada solamente a la retención de información en memoria de trabajo. En relación al desempeño en preguntas inferenciales, esto es de la información que no se encuentra explícita en el texto narrado-pero debe ser añadida durante la comprensión-, se observó tanto en la muestra total, como en la muestra de cinco y seis años por separado, que esta capacidad se encuentra asociada a la retención de información en memoria de trabajo y a la capacidad de sostener el foco de atención.

Respecto de los análisis de comparación entre los niños de 5 y 6 años, se observó en relación a las medidas de memoria de trabajo y atención sostenida, que los niños no difieren en su capacidad de memoria de trabajo, pero en cambio sí lo hacen en cuanto a su capacidad de sostener la atención. Los niños de 6 años tienen una mejor capacidad para poder sostener el foco de su atención sobre una tarea en comparación con los niños de 5 años. En cuanto a las medidas de comprensión de las historias narradas, se observó que los niños de 6 mostraron un mejor desempeño en la comprensión de información literal del cuento narrado, esto es de la información explícitamente mencionada durante la historia, y también un mejor desempeño en la respuesta 
a preguntas acerca del contenido inferencial; esto es, de elementos no mencionados pero requeridos para la comprensión. Este resultado es consistente con investigaciones previas en el área (Currie y Cain, 2015; Paris y Lindauer, 1976) que han mostrado desempeños mejores en niños de 6 años, al compararlos con niños de 5 años.

En cuanto al análisis de ecuaciones estructurales, en primer lugar, la diferencia en la edad de los participantes (analizada en meses) mostró explicar las diferencias en la capacidad de sostener la atención y las medidas obtenidas de comprensión. Este resultado es coherente con investigaciones previas acerca del desarrollo de las habilidades de comprensión (Kendeou et al., 2009; Lynch et al., 2008). En segundo lugar, la memoria de trabajo y la capacidad de sostener la atención, también explicaron las diferencias obtenidas en los puntajes de comprensión, resultado también coherente con otras investigaciones vinculadas al área de la comprensión a partir textos escritos (Cain, Oakhill y Bryant, 2004; Finney, Montgomery, Gillam y Evans, 2014; Van Dyke, Johns y Kukona, 2014), y también con investigaciones que han estudiado la comprensión de narraciones escuchadas (Currie y Cain, 2015; Strasser y del Rio, 2013) . De hecho, en la investigación de Currie y Cain (2015) se encontró un efecto directo y significativo de la memoria de trabajo sobre la generación de inferencias, y Strasser y del Rio (2013) encontraron un efecto directo de la memoria de trabajo en el recuerdo de historias $\mathrm{y}$ un efecto de la memoria de trabajo y de las habilidades ejecutivas (incluidas la capacidad de sostener la atención) en la comprensión de textos con imágenes, mediado a partir de habilidades de comprensión. En tercer lugar, el desarrollo madurativo medido a través de la edad no solo tiene una incidencia directa sobre la comprensión, sino que también tiene un efecto indirecto mediado por el desarrollo de habilidades cognitivas, en este caso la capacidad de sostener la atención.

En conjunto, los resultados de los análisis efectuados permiten indicar que la capacidad de la memoria de trabajo, esto es de retención y de manipulación de información, juega un rol importante en la comprensión de información literal del texto narrativo presentado de forma oral, como así también en la activación de información necesaria para la comprensión de dicha narración (inferencias). Aunque si bien la memoria de trabajo no muestra un salto en el desarrollo entre los 5 y los 6 años, como sí se observa en las medidas evaluadas de comprensión, las diferencias en la capacidad de los niños de esta habilidad repercuten en la comprensión. Respecto de la capacidad de poder sostener el foco de atención en una tarea, los análisis muestran diferencias respecto de la edad y asociaciones con respecto a las medidas de comprensión. Asimismo, el análisis de ecuaciones estructurales muestra que los menores tiempos en el desempeño de la tarea a realizar vinculados a un mejor rendimiento del foco atencional muestra un efecto, si bien menor que la memoria de trabajo, importante sobre la comprensión. Por lo tanto, el poder sostener el foco de atención en la tarea de comprensión es importante para la misma actividad. Este resultado es relevante para el ámbito educativo, ya que sería recomendable que la estructura académica preescolar incluya dentro de su currículo, actividades para el entrenamiento de habilidades atencionales y de memoria de trabajo, como puede ser la búsqueda visual de objetos, la búsqueda de una incoherencia en un texto breve y/o el recuerdo de historias. Además de actividades antes, durante y después de una historia, como puede ser, presentar palabras claves antes de contar la historia, llevar a cabo actividades de imaginería visual durante la historia y la realización de actividades que fomenten el recuerdo, el reconocimiento y la respuesta a preguntas sencillas de la narración.

Ciertas limitaciones del presente estudio deben tenerse en cuenta. La muestra ha sido constituida siguiendo un criterio no aleatorio por conveniencia, tomando las instituciones educativas que se ofrecieron a parti- 
cipar del estudio. Será sumamente relevante replicar estos datos en una muestra mayor y con características diferentes en cuanto al tipo de escuela de los participantes, el nivel lector de sus padres y ver el rol de la experiencia o frecuencia de exposición a textos narrativos narrados por parte de un adulto.

Futuras direcciones a partir del presente estudio podrían estudiar otras habilidades de comprensión de los niños prelectores, como ser el monitoreo de la comprensión y la comprensión de la estructura del texto. Asimismo, sería interesante estudiar cómo estas habilidades se vinculan con la capacidad de memoria de trabajo, la atención sostenida, el conocimiento previo sobre el texto y el conocimiento acerca del vocabulario, ya sea en cuanto a la amplitud o su profundidad.

\section{Referencias bibliográficas}

Arbuckle, J.L. (2014). IBM SPSS AMOS 22 User'S Guide. Mount Pleasant, SC: Amos Development Corporation.

Baddeley, A.D. (2010). Working Memory. Current Biology, 20(4), 136-140. http://dx.doi. org/10.1016/j.cub.2009.12.014

Barreyro, J.P., Cevasco, J., Burin, D.I. y Molinari, C. (2012). Working Memory Capacity and Individual Differences in the Making of Reinstatement and Elaborative Inferences. Spanish Journal of Psychology, 15(2), 471-479. http://dx.doi.org/10.5209/rev_ SJOP.2012.v15.n2.38857

Bernasconi, P. (2007). Hipo no nada.. Buenos Aires, AR: La Brujita de Papel.

Bourg, T., Bauer, P. y van den Broek, P. (1997). Building the bridges: The development of event comprehension and representation. En P. van den Broek, P. Bauer y T. Bourg (Eds.), Developmental spans in event comprehension and representation: Bridging fictional and actual events (pp. 385-407). Hillsdale, NJ: Erlbaum.

Browne, E. (1996). La sorpresa de Nandi.. Caracas, VE: Ediciones Ekaré.

Cain, K., Oakhill, J. y Bryant, P. (2004). Chil- dren's reading comprehension ability: Concurrent prediction by working memory, verbal ability, and component skills. Journal of Educational Psychology, 96, 31-42. http:// dx.doi.org/10.1037/0022-0663.96.1.31

Currie, N.K. y Cain, K. (2015). Children's inference generation: The role of vocabulary and working memory. Journal of Experimental Child Psychology, 137, 57-75. http://dx.doi. org/10.1016/j.jecp.2015.03.005

Diamond, A. y Goldman-Rakic, P.S. (1989). Comparison of human infants and rhesus monkeys on Piaget's tasks: Evidence of dependence on dorsolateral prefrontal cortex. Experimental Brain Research, 74(9), 24-40.

Finney, M.C., Montgomery, J.W., Gillam, R.B. y Evans, J.L. (2014). Role of Working Memory Storage and Attention Focus Switching in Children's Comprehension of Spoken Object Relative Sentences. Child Development Research, 2014, 1-11. http:// dx.doi.org/10.1155/2014/450734

Goldman, S.R. (1985). Inferential reasoning in and about narrative texts. En A.C. Graesser y J.B. Black (Eds.), The psychology of questions (pp. 247-276). Hillsdale, NJ: Lawrence Erlbaum Associates.

Gorman, B.K., Bingham, G.E., Fiestas, C.E. y Terry, N.P. (2016). Assessing the narrative abilities of Spanish-speaking preschool children: A Spanish adaptation of the narrative assessment protocol. Early Childhood Research Quarterly, 36, 307-317. http:// dx.doi.org/10.1016/j.ecresq.2015.12.025

Graesser, A.C. (2007). An Introduction to Strategic Reading Comprehension. En D. S. McNamara (Ed.), Reading Comprehension Strategies. Theories, Interventions, and Technologies (pp. 3-26). Mahwah, NJ: Lawrence Erlbaum Associates.

Graesser, A.C., Leon, J.A. y Otero, J.C. (2002). Introduction to the psychology of science text comprehension. En J. Otero, J.A. Leon y A.C. Graesser (Eds.), The psychology of science text comprehension (pp. 1-15). Mahwah, NJ: Erlbaum.

Graesser, A.C., Millis, K.K. y Zwaan, R.A. 
(1997). Discourse comprehension. Annual Review of Psychology, 48, 163-189. http:// dx.doi.org/10.1146/annurev.psych.48.1.163

Hu, L. y Bentler, P.M. (1998). Fit indices in covariance structure modeling: Sensitivity to underparameterized model misspecification. Psychological Methods, 3, 424-453. http:// dx.doi.org/10.1037/1082-989X.3.4.424

Hu, L. y Bentler, P.M. (1999). Cut-off criteria for fit indexes in covariance structure analysis: Conventional criteria versus new alternatives. Structural Equation Modeling, 6(1), 1-55. http://dx.doi. org/10.1080/10705519909540118

Irrazabal, N., Saux, G. y Burin, D. (2016). Formato de presentación, experticia y memoria de trabajo en la comprensión de instrucciones. Interdisciplinaria, 33(2), 215-229. http://dx.doi.org/ 10.16888/ interd.2016.33.2.2

Jung, R. (2009). El zorro y la Cigueña de Esopo.. Buenos Aires, AR: Heliasta.

Kaufman, A.S., y Kaufman, N.L. (1983). Batería de evaluación de Kaufmann para niños.. Madrid, ES: TEA Ediciones.

Kendeou, P., van den Broek, P., White, M.J. y Lynch, J.S. (2009). Predicting Reading Comprehension in Early Elementary School: The Independent Contributions of Oral Language and Decoding Skills. Journal of Educational Psychology, 101(4), 765-778. http://dx.doi.org/10.1037/a0015956

Kim, O., Kendeou, P., van den Broek, P., White, M.J. y Kremer, K. (2008). Cat, rat, and rugrats: Narrative comprehension in young children with Down syndrome. Journal of Developmental and Physical Disabilities, 20(4), 337-351. http://dx.doi.org/10.1007/ s10882-008-9101-0

Kintsch, W. (1998). Comprehension. A paradigm for cognition. Cambridge, MA: Cambridge University Press.

Kintsch, W. y van Dijk, T.A. (1978). Towards a model of text comprehension and production. Psychological Review, 85, 363-394. http:// dx.doi.org/10.1037/0033-295X.85.5.363

László, J. (2008). The science of stories: An intro- duction to narrative psychology. New York, NY: Routledge.

Leon, J.A. y Peñalba, G.E. (2002). Understanding Causality and Temporal Sequence in Scientific Discourse. In J. Otero, J.A. Leon, y A. Graesser (Eds.), The Psychology of Science Text Comprehension (pp. 155-178). Mahwah, NJ: Lawrence Erlabaum.

Lynch, J.S., van den Broek, P., Kremer, K.E., Kendeou, P., White, M.J. y Lorch, E.P. (2008). The Development of Narrative Comprehension and Its Relation to Other Early Reading Skills. Reading Psychology, 29(4), 327-365. http:// dx.doi.org/10.1080/02702710802165416

McKoon, G. y Ratcliff, R. (1992). Inference during reading. Psychological Review, 99, 440-466. http://dx.doi.org/10.1037/0033-295X.99.3.440

McVay, J.C. y Kane, M.J. (2012). Why does working memory capacity predict variation in reading comprehension? On the influence of mind wandering and executive attention. Journal of Experimental Psychology: General, 141(2), 302-320. http://dx.doi.org/10.1037/ a0025250

Molinari, C., Barreyro, J.P., Cevasco, J. y van den Broek, P. (2011). Generation of Emotional Inferences during Text Comprehension: Behavioral Data and Implementation through the Landscape Model. Escritos de Psicología, 4(1), 9-17. http://dx.doi.org/10.5231/psy. writ.2011.1803

Paris, S.G. y Lindauer, B.K. (1976). The role of inference in children's comprehension and memory for sentences. Cognitive Psychology, 8(2), 217-227. http://dx.doi.org/10.1016/00100285(76)90024-4

Paris, A.H. y Paris, S.G. (2003). Assessing narrative comprehension in young children. Reading Research Quarterly, 38(1), 36-76. http:// dx.doi.org/10.1598/RRQ.38.1.3

Rapp, D.N., van den Broek, P. y Kendeou, P. (2005). Integrating memory-based and constructionist processes in accounts of reading comprehension. Discourse Processes, 39, 299-316.

Sater, M., Givens, B. y Bruno, J.P. (2001). The Cognitive Neuroscience of sustained atten- 
tion: Where top-down meet bottom-up. Brain Research Reviews, 35, 146-160.

Silva, M. y Cain, K. (2015). The relations between lower and higher level comprehension skills and their role in prediction of early reading comprehension. Journal of Educational Psychology, 107(2), 321-331. http://dx.doi. org/10.1037/a0037769

Soprano, A.M. (2009). Cómo evaluar la atención $y$ las funciones ejecutivas en niños y adolescentes. Paidós: Buenos Aires, AR.

Stein, N.L. (1982). The definition of a story. Journal of Pragmatics, 6, 487-507.

Strasser, K. y del Río, F. (2013). The role of comprehension monitoring, theory of mind, and vocabulary depth in predicting story comprehension and recall of kindergarten children. Reading Research Quarterly, 49(2), 169-187. http://dx.doi.org/10.1002/rrq.68

Suh, S. y Trabasso, T. (1993). Inferences during reading: Converging evidence from discourse analysis, talk-aloud protocols, and recognition priming. Journal of Memory \& Language, 32, $279300 . \quad$ http://dx.doi.org/10.1006/ jmla.1993.1015

Villalonga Penna, M.M., Padilla Sabaté, C. y Burin, D. (2014). Relaciones entre decodificación, conocimiento léxico-semántico e inferencias en niños de escolaridad primaria. Interdisciplinaria, 31(2), 259-274. http://dx.doi.org/ 10.16888/interd.2014.31.2.5

van den Broek, P. (1989). Causal reasoning and inference making in judging the importance of story statements. Child Development, 60, 286-297. http://dx.doi.org/10.2307/1130976

van den Broek, P. (1994). Comprehension and memory of narrative texts: Inferences and coherence. In M.A. Gernsbacher (Ed.), Handbook of psycholinguistics (pp. 539-588). San Diego, CA: Academic Press.

van den Broek, P. (1997). Discovering the cements of the universe: The development of event comprehension from childhood to adulthood. In P. van den Broek, P. Bauer, y T. Bourg (Eds.),
Developmental spans in event comprehension: Bridging fictional and actual events (pp. 321342). Mahwah, NJ: Erlbaum.

van den Broek, P. y Kendeou, P. (2008). Cognitive processes in comprehension of science texts: The role of co-activation in confronting misconceptions. Applied Cognitive Psychology, 22, 335-351. http://dx.doi.org/10.1002/ acp. 1418

van den Broek, P., Kendeou, P., Kremer, K., Lynch, J.S., Butler, J., White, M.J. y Lorch, E.P. (2005). Assessment of comprehension abilities in young children. En S. Stahl y S. Paris (Eds.), Children's Reading Comprehension and Assessment (pp. 107-130). Mahwah, NJ: Erlbaum.

van den Broek, P., Risden, K.C. y Husebye-Hartmann, E. (1995). The role of readers' standards for coherence in the generation of inferences during reading. En R.F. Lorch y E.J. O'Brien (Eds.), Sources of coherence in reading (pp. 353-373). Hillsdale, NJ: Lawrence Erlbaum.

van den Broek, P., Rohleder, L. y Narváez, D. (1996). Causal inferences in the comprehension of literary text. In R.J. Kreuz \& M.S. MacNealy (Eds.), Empirical approaches to literature and aesthetics. Norwood, NJ: Ablex Publishing Corporation.

Van Dijk, T. y Kintsch, W. (1983). Strategies of discourse comprehension. New York, NY: Academic Press.

Van Dyke, J.A., Johns, C.L. y Kukona, A. (2014). Low working memory capacity is only spuriously related to poor reading comprehension. Cognition, 131(3), 373-403. http://dx.doi. org/10.1016/j.cognition.2014.01.007

Wechsler, D. (1998). Test de inteligencia para preescolares (WPPSI). [Pre-school intelligence test]. Buenos Aires, AR: Paidós.

Zwaan, R.A. y Singer, M. (2003). Text Comprehension. In A. Graesser, M.A. Gernsbacher, y S.R. Goldman (Eds.), Handbook of Discourse Processes (pp. 83-121). Mahwah, NJ: Lawrence Erlbaum Associates. 
